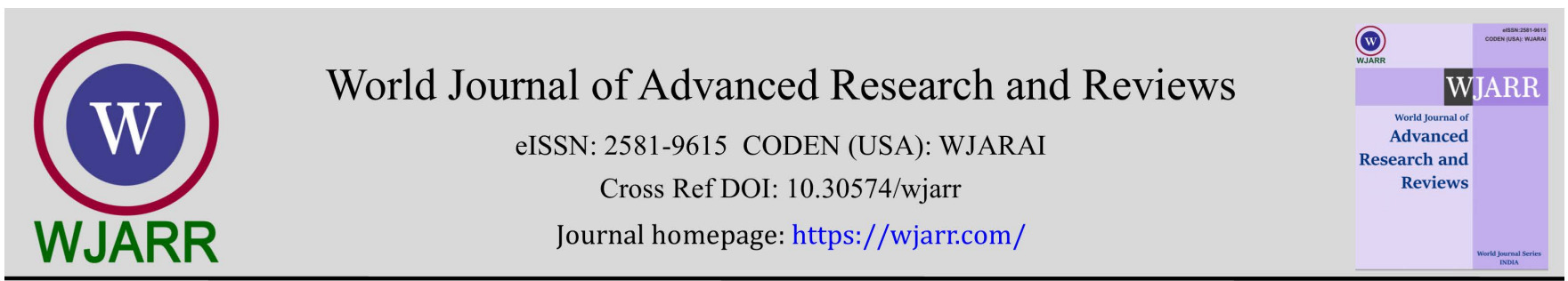

(RESEARCH ARTiClE)

\title{
The COVID-19 pandemic, associated mental health issues and music educational therapy; An international and Chinese approach
}

\author{
Wolfgang Mastnak* \\ Beijing Normal University BNU.
}

World Journal of Advanced Research and Reviews, 2022, 13(01), 543-551

Publication history: Received on 12 December 2021; revised on 17 January 2022; accepted on 19 January 2022

Article DOI: https://doi.org/10.30574/wjarr.2022.13.1.0057

\begin{abstract}
COVID-19 dynamics and measures to control the pandemic have worldwide caused a high amount of mental and affective disturbances. Particularly children and adolescents have been confronted with home-schooling, domestic violence, social isolation and loneliness, existential issues, as well as the experience of multifaceted panicmongering and related pathological reactions. Although estimated prevalence rates of COVID-19 associated mental disorders vary across studies, there is a statistical tendency suggesting that about one third of the younger population exhibits serious psychiatric issues. As this quantity goes beyond the capacity of institutionalised psychiatric care, new models combining education and therapy are needed and are challenging today's school systems. In Germany the COPSY study provided differential diagnostic data, and a music educational study used meta-synthetic methods to construct robust hypotheses which are considered stronger than usual hypotheses, but still need follow-up studies to explore underlying mechanisms and to estimate effect sizes. The related music-health-educational framework covers six key areas: (i) creative interaction to treat 'coronanxiety', (ii) focal listening to reduce symptoms of post-traumatic stress disorders, (iii) vocal immersion to improve psychosomatic self-regulation, (iv) sound scene improvisation to enhance selfactualisation and relieve learned helplessness, (v) artistic expression to support coping processes and communication skills and (vi) the 'Beloved Augustin phenomenon' concerning the positive impact of arts and joy on the immune system. Actually these approaches are being examined for application in China, alongside socio-cultural adaptations, qualitative research and neuroscientific studies on underlying mechanisms.
\end{abstract}

Keywords: Chinese music therapy; Culturally sensitive music therapy; Immune system; Pandemic; Music health education; Self-regulation

\section{Introduction}

The COVID-19 pandemic has significantly affected our minds and researchers even speak of an epidemiology of mental health problems in COVID-19 [1]. The global community is concerned about the pandemic and its long-term consequences, which impact on various spheres of life such as employment, freedom or family planning. And while national and international bodies such as the World Health Organization or regional ministries of health try to control the pandemic by identifying, testing and treating infected people, as well as behavioural measures and the promotion of vaccination, mental health problems associated with the whole COVID-19 situation have become a serious threat and public health challenge [2]:

The WHO has also expressed its concern over the pandemic's mental health and psycho-social consequences [...] It speculates that new measures such as self-isolation and quarantine have affected usual activities, routines, and livelihoods of people that may lead to an increase in loneliness, anxiety, depression, insomnia, harmful alcohol, and drug use, and self-harm or suicidal behavior [...] The lockdowns around the world have led to an increase in cases of domestic violence where women and

\footnotetext{
* Corresponding author: Wolfgang Mastnak

Beijing Normal University BNU.

Copyright (C) 2022 Author(s) retain the copyright of this article. This article is published under the terms of the Creative Commons Attribution Liscense 4.0.
} 


\begin{abstract}
children who live with domestic violence have no escape from their abusers during quarantine [...] Psychologists and mental health professionals speculate that the pandemic is going to impact on the mental health of the population globally with the increase in cases of depression, suicide, and self-harm, apart from other symptoms reported globally due to COVID 2019 [...] They speculate about the chances of developing neurotic disorders such as generalized anxiety disorder and obsessivecompulsive disorders (OCD) in large population groups [...] Besides mood-related and emotional outbursts especially panic, fear, avoidance and fear in meeting other people, fear of death (Thanatophobia), fear of getting isolated, stigmatization, fear of even not getting essential items, food, etc., may have its psychological manifestations [...] Interestingly the Coronavirus pandemic has another feature in this age of social media, where people are getting overloaded with rumors and misinformation which are not authentic and verified. Such rumors and unauthenticated information create fear, anxiety, and stress with a sudden and near-constant stream of news reports about an outbreak.
\end{abstract}

The complex conditions of the COVID-19 era have a considerably high impact on youth mental health and are particularly associated with depression and anxiety in adolescent cohorts [3]. Especially social isolation and loneliness have a negative impact on children and adolescents, who are probably more likely to experience high rates of depression and anxiety during and after enforced isolation ends [4].

While many international studies mainly focused on anxiety and depressive symptoms, German research tried to estimate the dynamics of long-term sequelae and to differentiate between distinct mental health issues in the younger generation. A prospective study from spring 2020 [5] suggested four dissimilar phases, (i) and acute phase, when children and adolescents experience the national or local breakout of COVID-19. Getting aware of measures to limit the spread of the pathogen and changes in the social atmosphere mark the acute phase and can trigger acute stress reaction and adjustment issues, insomnia, paranoid traits, disruptive behavioural attempts to cope with strains, 'scared stiff' and suicidal feelings; (ii) it follows the subacute phase including interferences of responsive habituation, ongoing anxiety, delusional ideas and signs of posttraumatic stress-disorders alongside their influence on developmental processes, personal growth and cognitive factors such as the power of concentration and achievement motivation; (iii) the posttraumatic phase, which follows on the subacute phase, is likely to entail, depending on the children's resilience or stress-susceptibility, pathological self-protective attitudes and personality features as well as mental issues such as posttraumatic stress disorders or depressive and avoidant personality traits; (iv) the late effect phase is expected after a relatively long symptom-free latency of several decades, and COVID-19-experiences afresh haunt an affected individual's mind and harm quality of life.

The both comprehensive and comparative COPSY study [6] investigated psychopathological impacts of the COVID-19 era on children and adolescents in Germany. The nationwide longitudinal survey was conducted with two waves during the pandemic and included children and adolescents aged 7 to 17 years. The self-report and parent-proxy surveys assessed HRQoL (KIDSCREEN-10), mental health problems (SDQ with the subscales emotional problems, conduct problems, hyperactivity and peer problems), anxiety (SCARED), depressive symptoms (CES-DC, PHQ-2) and psychosomatic complaints (HBSC-SCL). Mixed model panel regression analyses were conducted to examine longitudinal changes in mental health and to identify risk and resource factors:

A proportion of $30.9 \%$ suffered from mental health problems, such as conduct problems, hyperactivity, peer problems and emotional problems [...] the difference between pre-pandemic and wave 1 data was significant [...] but no significant difference was found comparing waves 1 and 2 [...] A proportion of $30.1 \%$ had symptoms of generalized anxiety [...] At wave 2 of the COPSY study, $63.0 \%$ had trouble concentrating [...] $61.9 \%$ had little interest or joy in activities [...] and 39.8\% felt sad [...] According to the PHQ-2, 15.1\% had depressive symptoms [...] Furthermore, children and adolescents reported psychosomatic complaints such as irritability (wave 2: 57.2\%, wave 1: 53.2\%, pre-pandemic: 39.8\%), headaches (wave 2: $46.4 \%$, wave 1: $40.5 \%$, pre-pandemic: $28.3 \%$ ), stomachaches (wave 2: $36.4 \%$, wave 1: $30.5 \%$, pre-pandemic: $21.3 \%$ ), and feeling low (wave 2: $43.4 \%$, wave 1: $33.8 \%$, pre-pandemic: $23.0 \%$ ).

The COVID-19 pandemic also affected the mental health of children and adolescents in China - and a cross-sectional study [7] which was conducted early after SARS-CoV-2 occurred in Wuhan suggested that nearly $40.4 \%$ of the sampled youth were found to be prone to psychological problems and $14.4 \%$ exhibited symptoms of post-traumatic stress disorder - and a study from the China Medical University in Shenyang [8] spoke of a significant psychosocial impact of the COVID-19 outbreak on children and adolescents: '22.28\% respondents were suffering from depressive symptoms. Seven significant factors associated with increased levels of anxiety, including female, resident in urban regions, emotion-focused coping style. Nine factors associated with increased levels of depression, such as smartphone addiction $[\ldots]$, Internet addiction $[\ldots]]^{\prime}$.

Although data vary, the occurrence of psychiatric issues in Chinese children and adolescents associated with the COVID19 pandemic is alarming and a cross-sectional study [9] among Chinese students aged 12-18 years focused explicitly on prevalence rates and socio-demographic correlates of depressive and anxiety symptoms affected by the outbreak of COVID-19: 


\begin{abstract}
A total of 8079 participants were involved in the study. An online survey was used to collect demographic data, assess students' awareness of COVID-19, and assess depressive and anxiety symptoms with the Patient Health Questionnaire (PHQ9) and the Generalized Anxiety Disorder (GAD-7) questionnaire, respectively. The prevalence of depressive symptoms, anxiety symptoms, and a combination of depressive and anxiety symptoms was $43.7 \%, 37.4 \%$, and $31.3 \%$, respectively, among Chinese high school students during the COVID-19 outbreak. Multivariable logistic regression analysis revealed that female gender was the higher risk factor for depressive and anxiety symptoms. In terms of grades, senior high school was a risk factor for depressive and anxiety symptoms; the higher the grade, the greater the prevalence of depressive and anxiety symptoms. Our findings show there is a high prevalence of psychological health problems among adolescents, which are negatively associated with the level of awareness of COVID-19. These findings suggest that the government needs to pay more attention to psychological health among adolescents while combating COVID-19.
\end{abstract}

As traumatisation such as associated with the COVID-19 pandemic tends to give rise to chronic mental and affective disorders as well as pathological personality traits, these issues will most probably not end with the end of the pandemic, hence the present study's intention to encourage long-term support of mental health in the Chinese younger population through music-based educational-therapeutic approaches.

\title{
2. Methods
}

A German study [10] used principles of systemic meta-syntheses to construct music-educational models for classroom education to alleviate mental and affective issues associated with the COVID-19 pandemic, as well as to enhance the students' resilience and arts-based self-regulation techniques.

Systemic meta-syntheses [11] are designed to construct new theories and to generate 'powered hypotheses', a process which can be considered complementary to the estimation of effect sizes as well as hypothesis-testing. In short: the various concepts of verification, power and truth matter. Akin to the concept of degrees of corroboration, the notion 'powered hypothesis' is based on epistemological strength: (i) similar to meta-analyses systemic meta-syntheses carefully select their components; different from meta-analyses, however, they are chosen because of their epistemological value and trans-disciplinary 'goodness of fit'; (ii) while the weight of a meta-analysis stems from the amount and methodological quality of the studies referred to, meta-syntheses depend on the quality of their linkage and how this blends with the inner logic of the entire framework; (iii) in line with the Gestalt-theoretical argument that the whole is greater than the sum of its parts, the theory of systemic meta-syntheses argues that the outcome of the whole study in not a simple and direct consequence of their parts, but substantially involves the mode of connecting its components and the epistemological rationale behind the synthesis, hence the term 'powered hypothesis', which also applies to the present study.

\section{Results}

The following music-based educational-therapeutic models were designed to alleviate COVID-19 associated psychiatric issues in primary and secondary school students in Germany. Assuming that pathological processes in German and Chinese children and adolescents are - with specific socio-cultural limitations - comparable, these form a basis for intended qualitative and inter-disciplinary research in China. Related in-service-training for teachers is intended.

\subsection{Coronanxiety and Creative Interactions}

'Coronanxiety' is an informal and meanwhile commonly, but differently used term. One definition concerns COVID-19 sufferers who are living with fear and helplessness such as patients in Quarantine who have developed negative feelings [12]: 'Stigma and uncertainty of viral disease progression are two main concerns expressed by COVID-19 patients. Patients have experienced significant psychological distress, especially during hospitalization [...] It is not only important to address the psychiatric symptoms for COVID19 patients, attention should be paid to patient perceived stigma and coping strategies when delivering psychological interventions'.

The other use of the term 'coronanxiety' is much broader and comprises all sorts of anxious feelings associated with the pandemic. By way of illustration, measures to control COVID-19, and particularly what had been experienced as panicmongering, caused a novel form of social anxiety with delusional thinking, an unshakable belief in the omnipresent inescapability of SARS-CoV-2-threats, culminating in the illusion of 'humanoid viruses': children who do not regard peers as possible friends, but mainly as contagious objects, hence their panic-like reactions. The features of coronanxiety vary considerably across individuals and encompass, for instance, existential fears as well as the feeling of being permanently haunted by the pandemic. 
Creative Interactions [13] is a multidisciplinary and intermodal concept of music education, particularly of elemental music education. It was founded by the Italian music educator and researcher Andrea Sangiorgio and focuses on the broad spectrum of creativity in interpersonal dynamics. The present model to treat coronanxiety combines creative processes, symbolic experience and principles of cognitive behavioural therapy (CBT). Although this approach is akin to exposure training and systemic desensitisation in CBT, it importantly involves creative empowerment and the mesmerising spirit of aesthetic experience.

\subsection{Acute or posttraumatic stress and focal listening}

There is robust evidence that prevalence rates of posttraumatic stress and psychological stress in the general population have been elevated due to COVID-19, and a meta-analysis [14] 'indicated that the pooled prevalence of posttraumatic stress symptoms and psychological stress in the general population was $23.88 \%$ and $24.84 \%$, respectively [...] Overall, nearly one-in-four adults experienced significant stress due to the COVID-19 pandemic. Psychological resources and services must be allocated to help address the mental health burden of COVID-19' - and studies on various stress disturbances in children and adolescents during the COVID-19 era suggested that these phenomena [15] 'shouldn't be overlooked in different populations as it is expected in a considerable proportion of children/adolescents with variable prevalence, risk factors and severity'.

A German study [16] on specific modes of music listening and their application to self-regulation of stress and burnout syndromes showed that a 'quasi selective attentiveness to perceived sound phenomena generates an experienced identity with the music listened to. This requires a certain ability of auditory concentration and might cause the temporary self-awareness of "being rhythm and sound" that virtually blinds out the consciousness of other dimensions of the self'. This form of listening is called 'focal listening' and can help to reduce acute stress as well as symptoms of posttraumatic stress disorder. In the present model, focal listening is suggested for classroom education to help students to alleviate stress experiences and stress-related issues - self-application of personalised methods as well as sustainable competence across the life-span are key objectives.

\subsection{Psychophysiological imbalance and vocal immersion}

Measures to control the pandemic have caused systemic imbalances, such as sociocultural or economic harm, alongside multiple risk factors related to the psychosocial and psychophysiological strain during the COVID-19 era [17]. In many medical disciplines and areas such as holistic medicine, complementary medicine or body-oriented forms of psychotherapy [18], theoretical frameworks assume a complex, dynamic and potentially fragile psychosomatic equilibrium. Its disturbance may lead to psychosomatic imbalance and associated symptoms. Consistent with these views, the present study assumes that, depending on individual psychosomatic vulnerabilities, the COVID-19 era has been likely to disturb this essential homeostasis and thus to bring about various syndromes. Consequently the goal is not to treat the syndromes themselves but to help to restore this homeostasis, hence the term 'psychosomatic rebalancing'.

Arts-based therapies provide a broad repertoire of relevant techniques, such as Sound Balancing, one of the four therapeutic areas of Sound Work [19]. The present study particularly suggests vocal immersion, a technique in which the practitioner focuses intensively on his/her voice, combining principles of concentrative and mindfulness meditation. Immersing oneself in deep meditative vocal practice, which considerably differs from conventional singing, may give rise to the experience of a harmonious union of sound and self. Although underlying mechanisms are still unclear, such self-regulation processes can greatly help to restore psychosomatic equilibrium alongside symptom reduction.

\subsection{Learned helplessness and creative self-actualisation}

Research about the influence of the COVID-19 pandemic on mental health mainly focused on anxiety, stress and depression, while the experience of helplessness seems to be - except for medical staff and trainees [20] - a nearly ignored problem. Informal qualitative investigations in Germany suggest that children and adolescents may exhibit symptoms of learned helplessness in relation to the COVID-19 situation, not only in the classical sense but also according to more recent approaches that involve anthropological, neuroscientific and evolutionary aspects [21]: 'The concept of learned helplessness defines an escape or avoidance deficit after uncontrollable stress and is regarded as a depressionlike coping deficit in aversive but avoidable situations. Based on a psychological construct, it ideally complements other stress-induced or genetic animal models for major depression. Because of excellent face, construct, and predictive validity, it has contributed to the elaboration of several pathophysiological concepts and has brought forward new treatment targets'. 
The present model suggests artistic creative forms of desensitisation as akin to standard models in cognitive behavioural therapy. Related processes are interconnected with self-actualisation, not only in Maslow's hierarchical sense as the highest level of psychological development after basic ego needs have been fulfilled, but - close to ancient wisdom of personal growth and Carl Roger's views [22] - as a most important factor of experienced identity:

Considerations of well-being or flourishing include Maslow's and Rogers' concepts of self-actualisation and actualising tendency. Recent empirical findings suggest that only a modest proportion of the population might be considered to be flourishing. Separate findings focused upon the nature and determinants of post-traumatic growth identify it as comparable to flourishing, and facilitated by supported accommodation to the trauma. This can be understood as reflecting self-actualisation. Empirical findings such as these provide ontological stability to a set of phenomena that share much with ancient teachings extolling redemption through suffering.

The present study suggests that creative artistic processes are a viable means to enhance self-actualisation, as well as experience of meaningful self-efficacy [23] to cope with learned helplessness, particularly when it has become a personality trait or a manifest pathological habit. In music educational contexts, sound scene improvisation is a typical approach involving the students' artistic talents to inspire self-actualisation as well as the experience of a deep and meaningful identity of the self and its performance.

Sound scene improvisation is a key model of Polyaesthetic Education [24], one of the best elaborated interdisciplinary schools of music education. Sound scene improvisation encourages students to design - through interactive creative processes - the plot of a music dramatic play, which includes various possibilities for improvisation and requires high aesthetic self-identity. Sound scene improvisation involves various psychodramatic principles [25] and is also used for psychopreventive and therapeutic purposes [26]. The present study combines music educational and arts-therapeutic approaches to soothe COVID-19 associated issues through sound scene improvisation.

\title{
3.5. Educational expressive therapy to support coping processes
}

In Europe, both political measures to control the pandemic and information policies have split the society. For instance, individuals who are reluctant to get vaccinated feel stigmatised and people are afraid of saying frankly what the think if it does not conform to the mainstream. Such dynamics impact on the ethical principles of tolerance as well as freedom of speech, and a certain silence of opinions 'goes viral', while at the same time riots against political steps to contol the pandemic have erupted [27]:

\begin{abstract}
The lived experience of the covid-19 pandemic has split Europe just as the euro and refugee crises did, with the south and the east feeling much more badly affected than the north and the west [...] The economic victims are more likely than others to say that restrictions have been too severe, and they tend to be more sceptical about their governments' intentions behind lockdowns. Europeans are divided over what they believe to be governments' motivations behind restrictions [...] Splits are appearing between those who believe that, in the context of the pandemic, the biggest threat to their freedom comes from governments, on the one hand, and those who fear the behaviour of their fellow citizens, on the other. There is a major generational divide, with the young more likely than the old to blame governments for the ongoing impact; the young also feel more badly affected [...] This also affects children and adolescents and may even inhibit their readiness to talk openly, especially about emotional problems related to the COVID-19 situation.
\end{abstract}

At this point we enter a vicious circle: speaking helps to cope with problems, while refusing to speak candidly may aggravate them and is likely to become a problem itself. Moreover, communication - particularly if individuals avoid talking about the true nature of an issue - tends to get dry, formal and distant. Habituation of such conversation styles risk to result in mental blocks affecting an individual's ability to express feelings and inner images.

Expressive arts therapies are frequently used in mindful and personalised paediatric care [28] as well as in other domains such as child oncology, psycho-oncology included [29]. Several key principles of expressive arts therapies resemble common music educational practices such as [30] music, dance, drawing, painting, sculpting or writing in a supportive setting to facilitate growth and healing. It is a process of discovering ourselves through any art form that comes from an emotional depth. It is not creating a "pretty" picture. It is not a dance ready for the stage. It is not a poem written and rewritten to perfection. We express inner feelings by creating outer forms. Expressive art refers to using the emotional, intuitive aspects of ourselves in various media'.

In this sense, the present article recommends the use of expressive arts in music classroom-education to support interpersonal flow of communication, the ability to express inner processes through artistic media and thus to facilitate coping with COVID-19 associated mental and affective issues. 


\subsection{The Beloved Augustin phenomenon}

The Beloved Augustin, in German language 'Der liebe Augustin' - his real name was Markus Augustin - lived in the $17^{\text {th }}$ century in Vienna. It was the time of the 'Great Plague' caused by the 'Black Death bacteria' [31] - during the COVID-19 pandemic researchers pointed out that also ancient times were confronted with pandemics [32]. One day of the year 1679 he, who was well known as a gregarious street musician brimming with humour, was found, seemingly dead, and thrown into a plague pit near the church St. Ulrich (today $7^{\text {th }}$ district of Vienna). However, Augustin was not dead, just tremendously drunk. The next day he woke up in the mass grave, began to play his bagpipes - and was saved.

Referring to the story of the Beloved Augustin we have to emphasise that there is high evidence that the threats of the COVID-19 pandemic cannot be compared with the horror of the Black Death, and Richard Charles Horton [33] from the Lancet stated:

\footnotetext{
All of our interventions have focused on cutting lines of viral transmission, thereby controlling the spread of the pathogen. The "science" that has guided governments has been driven mostly by epidemic modellers and infectious disease specialists, who understandably frame the present health emergency in centuries-old terms of plague. But what we have learned so far tells us that the story of COVID-19 is not so simple. Two categories of disease are interacting within specific populationsinfection with severe acute respiratory syndrome coronavirus 2 (SARS-CoV-2) and an array of non-communicable diseases (NCDs). These conditions are clustering within social groups according to patterns of inequality deeply embedded in our societies. The aggregation of these diseases on a background of social and economic disparity exacerbates the adverse effects of each separate disease. COVID-19 is not a pandemic. It is a syndemic. The syndemic nature of the threat we face means that a more nuanced approach is needed if we are to protect the health of our communities.
}

Discussing COVID-19 vulnerabilities involves the question how to improve our immune response, and the enigma of Augustin's enormous immune capacity comes into play. The Harvard Medical School [34] posted recommendations how to strengthen our immune system to fight off diseases and highlighted the importance of healthy lifestyles including regular exercises and minimising stress. Moreover, Yoram Barak [35] discussed the positive influence of happiness on the immune system: 'an increase in secretory immunoglobulin A and a decrease in salivary cortisol were induced by pleasant emotions [...] There is data to support the hypothesis that individuals characterized by a more negative affective style poorly recruit their immune response and may be at risk for illness more so than those with a positive affective style'.

Discussing auditory stimuli and their impact on the immune system, a study from the Shanghai Jiao Tong University School of Medicine [36] pointed out that 'in humans, both innate immunity and specific immunity can be influenced by noise, and different exposure durations and intensities of noise may exert various effects on the immune system. Shortterm or low-intensity noise can enhance immune function, while long-term or high-intensity noise suppresses it'. Additionally, the authors explained that 'some studies have shown that music can improve immune function and alleviate the adverse effects caused by noise'.

The Beloved Augustin may serve as a symbol for boosting one's immune system: he loved his live and seemed to be free of stress and anxieties, he enjoyed the invigorating power of singing and playing music alongside his individual form of endurance training: walking through Vienna day and night. His live can be regarded as a maximum of self-actualisation and self-efficacy, far beyond external manipulation and heteronomy.

\section{Conclusion \& Perspectives}

Although the present study is based on systemic meta-syntheses and thus designed to yield robust hypotheses, there is still the need of further research: qualitative approaches that shall reveal the nature and characteristics of psychophysiological responses to educational-therapeutic models, and quantitative ones which shall help to estimate their effect sizes.

The present models were developed in Germany and relate to mental issues of German children and adolescents. But how can we apply them to the Chinese youth? Or how must they get tailored to the needs of affected individuals with Han-acculturation or ethnic minority backgrounds? These questions go hand in hand with key issues of culturally sensitive music therapy.

Although these models shall help to alleviate specific symptoms and syndromes which stem from adverse experiences and maladaptation during the COVID-19 era, they also evoke genuine music educational spirit and involve music educational values such as refining individual aesthetic experience. They shall also encourage further studies about (implicit) therapeutic effects of music educational models, as well as music educational effects of typical music 
therapeutic approaches. Such studies have also inspired the term 'clinical music education' and encompass notions such as the 'aesthetic self', a key topic of the aesthetic education research group of the Shanghai Conservatory of Music.

In many countries and among various social circles there is the erroneous assumption that the end of the pandemic will coincide with the disappearance of COVID-19 associated mental issues. From a psychopathological and psychiatric perspective, however, we have to take the possibility of perseveration, chronification and latency into account. There is a realistic probability that music education and school system will have to change. This calls for interdisciplinary research and cross-cultural collaboration. Beijing Normal University could serve as an international platform.

\section{Compliance with ethical standards}

\section{Acknowledgments}

This research received no specific grant from any funding agency in the public, commercial or not-for-profit sectors.

\section{Disclosure of conflict of interest}

The author declares no conflict of interest.

\section{Statement of ethical approval}

No approval is needed for this type of research.

\section{References}

[1] Hossain MM, Tasnim S, Sultana A, Faizah F, Mazumder H, Zou L, McKyer ELJ, Ahmed HU, Ma P. Epidemiology of mental health problems in COVID-19: a review. F1000Research. 2020; 9: 636.

[2] Kumar A, Nayar KR. COVID 19 and its mental health consequences. Journal of Mental Health. 2021; 30(1): 1-2.

[3] Nearchou F, Flinn C, Niland R, Subramaniam SS, Hennessy E. Exploring the impact of COVID-19 on mental health outcomes in children and adolescents: a systematic review. International Journal of Environmental Research and Public Health. 2020; 17(22): 8479.

[4] Loades ME, Chatburn E, Higson-Sweeney N, Reynolds S, Shafran R, Brigden A, Linney C, McManus MN, Borwick C, Crawley E. Rapid systematic review: the impact of social isolation and loneliness on the mental health of children and adolescents in the context of COVID-19. Journal of the American Academy of Child and Adolescent Psychiatry. 2020; 59(11): 1218-1239.e3.

[5] Mastnak W. Psychopathological problems related to the COVID-19 pandemic and possible prevention with music therapy. Acta Paediatrica. 2020; 109(8): 1516-1518.

[6] Ravens-Sieberer U, Kaman A, Erhart M, Otto C, Devine J, Löffler C, Hurrelmann K, Bullinger M, Barkmann C, Siegel NA, Simon AM, Wieler LH, Schlack R, Hölling H. Quality of life and mental health in children and adolescents during the first year of the COVID-19 pandemic: results of a two-wave nationwide population-based study. European Child \& Adolescent Psychiatry. 2021; 12: 1-14.

[7] Liang L, Ren H, Cao R, Hu Y, Qin Z, Li C, Mei S. The effect of COVID-19 on youth mental health. Psychiatric Quarterly. 2020; 91(3): 841-852.

[8] Duan L, Shao X, Wang Y, Huang Y, Miao J, Yang X, Zhu G. An investigation of mental health status of children and adolescents in china during the outbreak of COVID-19. Journal of Affective Disorders. 2020; 275: 112-118.

[9] Zhou SJ, Zhang LG, Wang LL, Guo ZC, Wang JQ, Chen JC, Liu M, Chen X, Chen JX. Prevalence and socio-demographic correlates of psychological health problems in Chinese adolescents during the outbreak of COVID-19. European Child \& Adolescent Psychiatry. 2020; 29(6): 749-758.

[10] Mastnak W. Psychopathologische Folgen der COVID-19-Ära. Eine Herausforderung für die Musikpädagogik? Diskussion Musikpädagogik. 2021; 92(4): 4-11.

[11] Mastnak W. Systemic Meta-Synthesis. ResearchGate. 2021.

[12] Sonawane S, Sharma H, Tetarbe T. Coronanxiety. Journal of Preventive Medicine and Holistic Health. 2020; 6(2): 52-53. 
[13] Sangiorgio A, Mastnak W, eds. Creative Interactions. Dynamic processes in group music activities. Munich: University of Music and Performing Arts. 2020.

[14] Cooke JE, Eirich R, Racine N, Madigan S. Prevalence of posttraumatic and general psychological stress during COVID-19: A rapid review and meta-analysis. Psychiatry Research. 2020; 292: 113347.

[15] Sayed MH, Hegazi MA, El-Baz MS, Alahmadi TS, Zubairi NA, Altuwiriqi MA, Saeedi FA, Atwah AF, Abdulhaq NM, Almurashi SH. COVID-19 related posttraumatic stress disorder in children and adolescents in Saudi Arabia. PLoS One. $2021 ; 16(8)$ : e0255440.

[16] Mastnak W, Köhler-Massinger W. Modes of music listening to modulate stress and prevent burnout. Musik-, Tanzund Kunsttherapie. 2017; 27(2): 123-128.

[17] Clemente-Suárez VJ, Dalamitros AA, Beltran-Velasco AI, Mielgo-Ayuso J, Tornero-Aguilera JF. Social and psychophysiological consequences of the COVID-19 pandemic: an extensive literature review. Frontiers in Psychology. 2020; 11: 580225.

[18] Ehrenfreund H. The disturbance of the psychosomatic balance. In: Payne H, Koch S, Tantia J, Fuchs T, eds. The Routledge International Handbook of Embodied Perspectives in Psychotherapy. London: Routledge; $2019 ; 17$.

[19] Mastnak W. Community Sound Work: Music in open health settings - voice body, inclusion and therapy, individuality and indication. International Journal of Community Music. 2016; 9(1): 49-63.

[20] Shaw SCK. Hopelessness, helplessness and resilience: The importance of safeguarding our trainees' mental wellbeing during the COVID-19 pandemic. Nurse Education in Practice. 2020; 44: 102780.

[21] Vollmayr B, Gass P. Learned helplessness: unique features and translational value of a cognitive depression model. Cell and Tissue Research. 2013; 354(1): 171-178.

[22] Middleton H. Flourishing and posttraumatic growth. An empirical take on ancient wisdoms. Health Care Analysis. 2016; 24(2): 133-147.

[23] Sheeran P, Maki A, Montanaro E, Avishai-Yitshak A, Bryan A, Klein WM, Miles E, Rothman AJ. The impact of changing attitudes, norms, and self-efficacy on health-related intentions and behavior: A meta-analysis. Health Psychology. 2016; 35(11): 1178-1188.

[24] Allesch C, Schwarzbauer M. Polyaesthetic Education: A model for integrating cultural experiences. In: Erzen N, ed. Congress Book II. Selected Papers XVIIth International Congress of Aesthetics. Ankara: Sanart. 2009; 19-27.

[25] Mastnak W. Psychodrama und Klangszenenimprovisation. In: Roscher W, ed. Sinn und Widerspruch musikalischer Bildung. Beiträge zu „poiesis“ und „aisthesis“ heute. München, Salzburg: Katzbichler. 1993; 105130 .

[26] Mastnak W. Klangszenenimprovisation in Psychoprävention \& Therapie. Musik-, Tanz- und Kunsttherapie. 2011; 22(4): 1-12.

[27] Krastev I, Leonard M. Europe's invisible divides: How covid-19 is polarising European politics. 2021.

[28] Diamond S, Lev-Wiesel R. The title "therapy" and what do you do with it as a child? Recollections of being in child expressive arts group therapy. Clinical Child Psychology and Psychiatry. 2017; 22(1): 152-164.

[29] Raybin JL, Barr E, Krajicek M, Jones J. How does creative arts therapy reduce distress for children with cancer? A metasynthesis of extant qualitative literature. Journal of Pediatric Oncology Nursing. 2020; 37(2): 91-104.

[30] Rogers N. The path to wholeness: person-centered expressive arts therapy. Psychotherapy.net. 2022.

[31] Barbieri R, Signoli M, Chevé D, Costedoat C, Tzortzis S, Aboudharam G, Raoult D, Drancourt M. Yersinia pestis: the natural history of Plague. Clinical Microbiology Reviews. 2020; 34(1): e00044-19.

[32] Morens DM, Daszak P, Markel H, Taubenberger JK. Pandemic COVID-19 joins history's pandemic legion. mBio. 2020; 11(3): e00812-20.

[33] Horton R. Offline: COVID-19 is not a pandemic. Lancet. 2020; 396(10255): 874.

[34] Harvard Medical School. How to boost your immune system. Harvard Health Publishing. 2021.

[35] Barak Y. The immune system and happiness. Autoimmunity Reviews. 2006; 5(8): 523-527.

[36] Zhang A, Zou T, Guo D, Wang Q, Shen Y, Hu H, Ye B, Xiang M. The immune system can hear noise. Frontiers in Immunology. 2021; 11: 619189. 